\title{
Optimización de Consumo Energético de un Modelo de Diseño \\ Paramétrico
}

\section{Energy Consumption Optimization of a Parametric \\ Design Model}

\author{
Patricia Edith Camporeale* \\ Laboratorio de Arquitectura y Hábitat Sustentable (LAyHS FAU UNLP). \\ layhs@fau.unlp.edu.ar \\ Jorge Daniel Czajkowski* \\ Laboratorio de Arquitectura y Hábitat Sustentable (LAyHS FAU UNLP)
}

* Doctora en Arquitectura y Urbanismo por la UNLP. Doctora en Arquitectura por la Universidad de Sevilla. Mi área experta es la optimización del diseño arquitectónico y urbano a través de la minimización de la demanda energética de la envolvente edilicia, la energía incorporada de los materiales y los residuos de demolición y construcción, en el marco del Ciclo de Vida del edificio. Publiqué trabajos en revistas científicas del primer cuartil (Q1, JCR) y participé de numerosas conferencias en el país y en el exterior presentando mis trabajos.

** Jorge Daniel Czajkowski es Arquitecto, Profesor titular de Instalaciones I-II, Director del Laboratorio de Arquitectura y Hábitat Sustentable en la Facultad de Arquitectura y Urbanismo de la Universidad Nacional de La Plata. Es Investigador Independiente del Consejo Nacional de Investigaciones Científicas y Técnicas. Mail: jdczajko@gmail.com, czajko@ing.unlp.edu.ar 


\section{Resumen}

Este trabajo está enmarcado en un proyecto de investigación más amplio sobre la optimización del diseño bioambiental paramétrico. Se determina una envolvente paramétrica eficiente, que satisfaga la ley 4458/12 de Normas de Acondicionamiento Térmico en la Construcción de Edificios de la Ciudad de Buenos Aires y las Normas IRAM 11604 (coeficiente volumétrico admisible de calefacción) y 11659/1-2 (coeficiente volumétrico admisible de refrigeración), incorporando variables bioambientales: cargas térmicas solares, de la envolvente y de la ocupación. Se aplican índices bioclimáticos de calefacción y refrigeración diseñados previamente, obteniendo diferentes alternativas edilicias de desempeño energético equivalente y configuración espacial diferenciada. Luego, se elige una alternativa y se optimiza la máxima superficie vidriada con el menor consumo energético. Se busca encontrar un equilibrio entre las ganancias y las pérdidas térmicas, al mismo tiempo que evitar el sobrecalentamiento, con la incorporación de dispositivos pasivos. Se concluye que puede considerarse válida la optimización, a través de un proceso paramétrico, porque muestra el desempeño energético de la envolvente en tiempo real. De esta manera, disminuyen los costos en horas de trabajo del diseñador.

Palabras clave: índices bioambientales; optimización consumo energético.

\section{Abstract}

This paper is part of a wider work on energy consumption optimization in parametric bioclimatic design. First, we determine an efficient parametric envelope, which fulfills Law 4458/12 on Thermal Conditioning Standards for Buildings in Buenos Aires City. The envelope also satisfies IRAM 11604 Standard on Admissible Volumetric Coefficient in Heating and IRAM 11659/1 -2 Standard on Admissible Volumetric in Cooling, while adding bioclimatic variables: solar, occupancy and envelope thermal loads. We apply bioclimatic indexes in heating and cooling, which have been previously designed. We obtain building alternatives with equivalent energy-performance but different morphology. Then, we choose an alternative and optimize maximum fenestration with minimum energy consumption. We look for equilibrium between solar gains, thermal losses, as long as we avoid overheating by means of passive devices. We can conclude that parameterization allows control energy performance of the envelope in real time, lowering costs on designer hours.

Keywords: bioclimatic indexes; energy consumption optimization 


\section{Introducción}

La demanda de energía en las ciudades- que se expanden con la migración de la población rural- no deja de incrementarse, aumentando las emisiones gases de efecto invernadero (GEI), que provocan el Cambio Climático. Sabemos quelos edificios consumen más de un tercio de la energía primaria generada en nuestro país (BEN 2013), por lo cual, es imprescindibleincorporar el concepto de eficiencia energética desde el proceso de diseño arquitectónico.

El diseño paramétrico se ha introducido en la disciplina para revolucionar la proyectación arquitectónica (Terzidis, 2006). Mediante la parametrización, se pueden aplicar herramientas digitales en un proceso transparente y en tiempo real (Leach, 2004). Junto con estas características, se incorpora la materialidad edilicia, lo cual nos permite, a su vez, evaluar la performance energética del mismo. El diseño bioambiental paramétrico es el que aúna la parametrización con la sostenibilidad, poniendo el énfasis en la eficiencia energética (Peronato, 2015).

Esta herramienta es un aporte para las primeras instancias del proyecto, cuando se toman aquellas decisiones que influirán a lo largo de todo su desarrollo: desde el proyecto, pasando por la construcción hasta el fin de la vida útil del edificio. En la etapa de operación y mantenimiento del edificio es cuando se registran los mayores gastos en acondicionamiento térmico.

En este trabajo, hemos desarrollado un edificio en altura de usos variados. Esta tipología es frecuente en la praxis profesional. Se pretende generar una reflexión en el campo de la investigación proyectual a través del uso de herramientas digitales que evalúan la performance energética, al mismo tiempo que su morfología y materialidad (Iqbal, 2006).

\section{Metodología}

Se parametriza un edificio en altura de oficinas, vivienda y uso mixto, dividido en tres volúmenes de diferente altura, agrupados en una morfología integrada (Tabla 1).

Está situado en un terreno urbano de $50 \mathrm{~m} \times$ $50 \mathrm{~m}$ rodeado de 3 calles con orientación Norte y costa de río sobre el lado Este. La zona bioambiental correspondiente es la Illb (templado cálido húmedo) del área metropolitana de Buenos Aires (AMBA) (IRAM 11603) (Figura1).

Luego de definida la ubicación del lote, se aplican las restricciones previstas por el Código Ambiental de la Ciudad de Buenos Aires, respetando la ocupación del terreno (60\% sup. parcela), el máximo volumen construible de la parcela (470\% de la sup. parcela) y los retiros perimetrales de $3 \mathrm{~m}$ y de fondo de $5 \mathrm{~m}$.

Con esta información, se definen paramétricamente los volúmenes que componen el edificio, el porcentaje de superficie vidriada por fachada y la materialidad de la envolvente (Figura 2), a través del programa de modelado en 3D, Rhinoceros $5 \AA$, y su plug-in de diseño paramétrico Grasshopper ${ }^{\circledR}$.

Tabla 1.Usos y áreas. Fuente: Elaboración propia

\begin{tabular}{|l|l|l|l|l|}
\hline Usos & $\begin{array}{l}\mathbf{n}^{\circ} \\
\text { plantas }\end{array}$ & $\begin{array}{l}\text { superficie por } \\
\text { planta }\end{array}$ & planta & H volumen \\
\hline & $\mathrm{u}$ & $\mathrm{m}^{2}$ & $\mathrm{~m}$ & $\mathrm{~m}$ \\
\hline vivienda & 10 & 300 & 2,75 & 27,5 \\
\hline oficina & 20 & 250 & 2,75 & 55 \\
\hline mixto & 15 & 250 & 2,75 & 41,25 \\
\hline sup. total & & 11750 & & \\
\hline
\end{tabular}




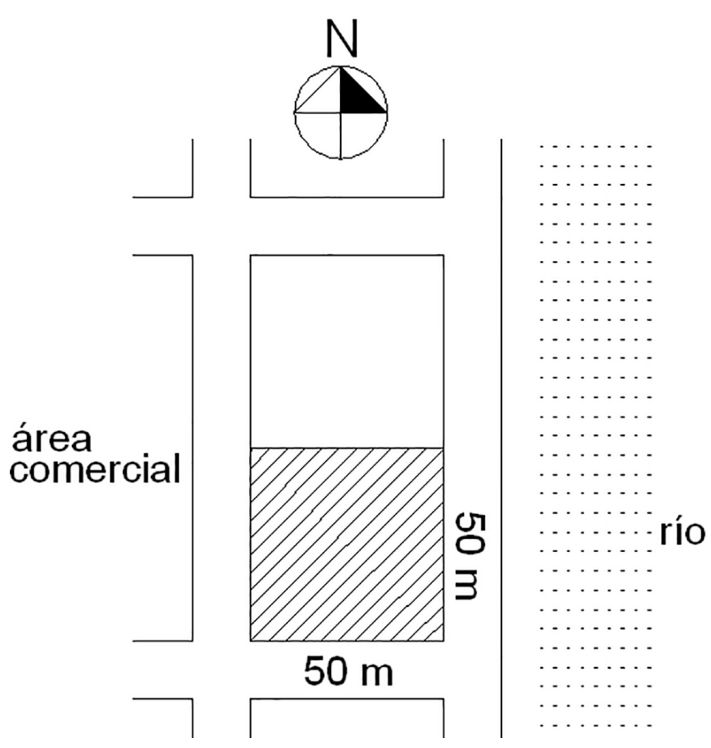

Tabla 2.Transmitancia térmica de la envolvente Fuente: los autores

\begin{tabular}{|c|c|c|}
\hline $\mathbf{k}$ & proyecto & adm. nivel B \\
\hline $\begin{array}{c}\text { muros (invierno y } \\
\text { vernano) }\end{array}$ & $0.71 \mathrm{~W} / \mathrm{m} 2 \mathrm{~K}$ & $1 / 1.25$ \\
\hline cubiertas (invierno) & $0.51 \mathrm{~W} / \mathrm{m} 2 \mathrm{~K}$ & 0.83 \\
\hline cubiertas (verano) & $0.46 \mathrm{~W} / \mathrm{m} 2 \mathrm{~K}$ & 0.83 \\
\hline k ventanas & $2.61 \mathrm{~W} / \mathrm{m} 2 \mathrm{~K}$ & 4 \\
\hline k piso & $1.08 \mathrm{~W} / \mathrm{ml}$ & noaplic. \\
\hline
\end{tabular}

La envolvente cumple con las normas IRAM de confort higrotérmico 11507-4 y 11605. El muro desde el exterior hacia el interior se compone de $0,025 \mathrm{~m}$ de poliestireno expandido revocado con muro de ladrillo hueco de 0,18m y terminación interior en yeso, las cubiertas constan de una membrana asfáltica de aluminio con 0,03m de espuma de poliuretano in situ, losa alivianada de hormigón pretensado con bloques depoliestireno expandido y cielorraso suspendido de yeso (Camporeale et al, 2015). Las ventanas son de doble vidriado hermético de $6 \mathrm{~mm}$ con cámara de aire de $12 \mathrm{mmcon}$ marco de aluminio y ruptura de puente térmico. El factor de protección solar es de 0,35. La transmitancia térmica de los elementos que componen la envolvente puede observarse en la Tabla 2.

Las superficies vidriadas se distribuyen de la siguiente manera:

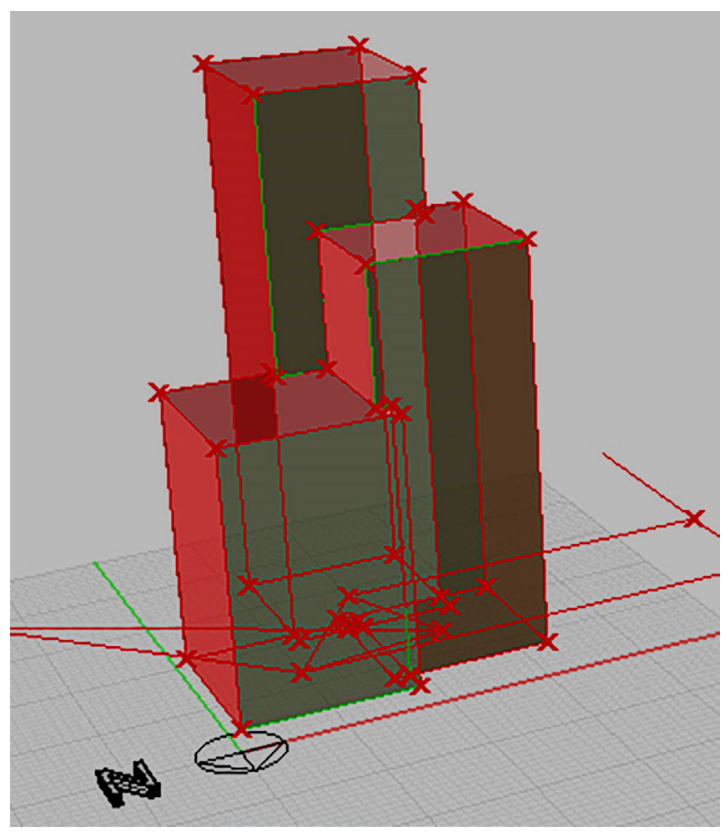

- Norte: $50 \%$

- Sur\%: $10 \%$

- Este: $40 \%$

- Oeste: $30 \%$

A continuación, ingresamos los coeficientes de temperatura horaria (Gonzalo, 1998), la máxima y la mínima y el programa calculalas temperaturas horarias para un día típico de invierno y otro de verano. Las temperaturas máximas y mínimas se obtienen del promedio ponderado de los meses de julio y enero de los años 2009 a 2012 inclusive, de datos recabados por la estación meteorológica que nuestro laboratorio posee en la localidad de Gonnet, partido de La Plata. Para el cálculo de consumo energético, utilizamos la temperatura sol-aire a los efectos de considerar la incidencia de la radiación solar sobre la envolvente. Calculamos a través de nuestro programa, asimismo, la infiltración del aire en las carpinterías, con un incremento de la velocidad media del viento $3,9 \mathrm{~m} / \mathrm{s}$ a $10 \mathrm{~m}$ de altura) proporcional a la altura del edificio (IRAM 11603).

Con estos datos (envolvente, temperaturas, radiación solar, infiltración), confeccionamos los índices de consumo energético Bcal (Ec. 1) y Bref (Ec. 2)en W/m3.día, basados en la fórmula de Gref (IRAM 11659 1-2) 


$$
B_{c a l}=\frac{1}{V} \cdot \sum_{i=1}^{n}\left(Q_{c}^{\prime}+Q_{a}^{\prime}-Q_{s}^{\prime}-Q_{o}^{\prime}\right)
$$

siendo:

Q'c= pérdidas térmicas por conducción de la envolvente: muros, ventanas, cubiertas (W):

Q'a= pérdidas térmicas por infiltración (W)en ventanas:

Q's= ganancias solares a través de las ventanas (W)

Q'o= ganancias internas por ocupación y equipamiento $(\mathrm{W})$

y

$B_{r \in f}=\frac{1}{V} \cdot \sum Q_{c}^{\prime}+Q_{a}^{\prime}+Q_{s}^{\prime}+Q_{o}^{\prime}$

siendo:

Q'c= ganancias térmicas por conducción dela envolvente: muros, ventanas y cubiertas(W)

Q'a= ganancias térmicas por infiltración en ven$\operatorname{tanas}(\mathrm{W})$

Q's= ganancias solares a través delas ventanas (W)

Q'o= ganancias internas por ocupación y equipamiento $(\mathrm{W})$

Luego el programa itera mediante un algoritmo genético (AG)provisto por Grasshopper®:Galapagos,(Mitchell, 1998). El AG busca dentro de una población de soluciones morfológicas posibles (Goldberg, 1997)minimizando el consumo energético de verano (Bref) que es sustancialmente superior al de invierno (Bcal) para la zona bioambiental donde se realiza el experimento.

Esta optimización de la morfología edilicia para minimizar Bref,se realiza con un factor solar de 0,35 . Calculamos asimismo Bcal para la hora 14, con el objeto de investigar si existe sobrecalentamiento. Tomamos luego la alternativa morfológica $1 \mathrm{y}$, manteniendo el valor de Brefobtenido, iteramos con el AG, no ya sobre la morfología, sino sobre los porcentajes de elementos vidriados en cada fachada, incrementando el factor solar a 0,10. Para alcanzar nuestro objetivo, mejoramos los dispositivos de protección solar, colocando cortinas venecianas externas de aluminio, con un factor de protección solar (Fs) igual a 0.10 , proporcionado por el fabricante ${ }^{1}$.

1. http://www.hunterdouglas.com/horizontal-blinds/naturalelements

\section{Resultados}

Una vez finalizada la carga de datos parametrizados, se corre el algoritmo genético hasta obtener soluciones edilicias optimizadas. Las dieciocho alternativas edilicias de mejor desempeño energético se muestran en la Figura 3.Seleccionamos aquéllas con menor consumo para verano, siendo este último muy superior al de invierno (el consumo para calefacción es muy inferior cuando se tiene una envolvente bastante eficiente). La superficie de la envolvente de cada alternativa tienen una variación del 23.4\%, dependiendo de cada configuración. Otro tanto ocurre con la superficie cubierta de las alternativas, que presentan una variación del $6.5 \%$, y con el volumen efectivo, cuya variación es 11.65\% (Tabla 3).

Los consumos de cada alternativa se expresan en la Tabla 3, donde se muestra el consumo para un día típico de verano y uno de invierno, la superficie de la envolvente, la superficie cubierta y el volumen efectivo de cada alternativa. Es notable la diferencia entre ambos consumos, calefacción y refrigeración, ya que la envolvente eficiente cubre gran parte de la demanda de energía para calefacción.

Comparando la incidencia de la relación porcentual vidrio/muro y los coeficientes BcalyBref de las alternativas obtenidas, obtenemos la dispersión que se muestra en la Figura 4

Figura 3. Vista NO de las alternativas obtenidas Fuente: elaboración propia

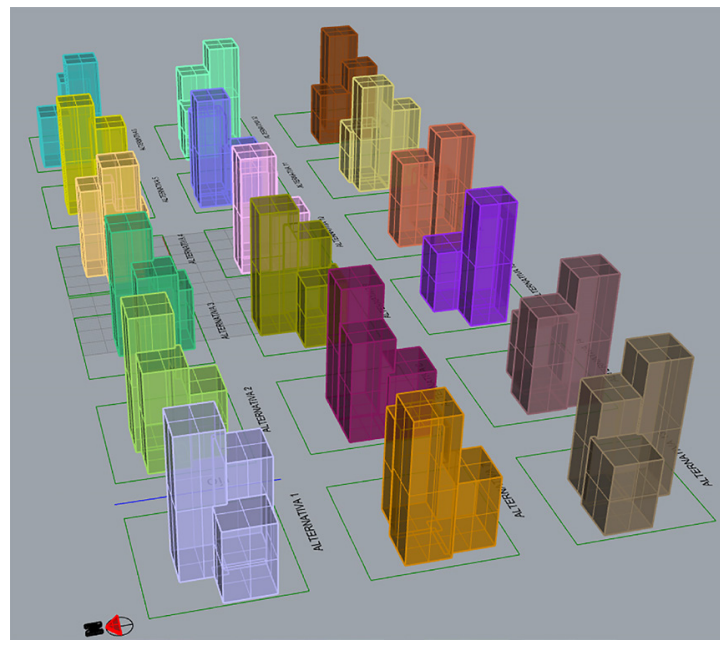


Tabla 3. Índices Bcal y Bref Fuente: elaboración propia

\begin{tabular}{|c|c|c|c|c|c|c|}
\hline $\begin{array}{c}\text { alternativa } \\
\cdot\end{array}$ & $\begin{array}{c}\text { sup. } \\
\text { envolvent } \\
e\end{array}$ & $\begin{array}{c}\text { sup. } \\
\text { cubierta } \\
\text { edificio }\end{array}$ & $\begin{array}{c}\text { vol. efect. } \\
\text { edificio }\end{array}$ & $\mathrm{B}_{\text {ref }}$ & $\mathrm{B}_{\text {cal }}$ & $\begin{array}{c}\% \\
\text { vidrio/opac } \\
\circ\end{array}$ \\
\cline { 2 - 6 } & $\mathrm{m}^{2}$ & $\mathrm{~m}^{2}$ & $\mathrm{~m}^{3}$ & $W / \mathrm{m}^{3}$. dia & W/m $\mathrm{m}^{3}$. dia & \\
\hline 1 & 4689,76 & 11046,15 & 27615,37 & 326,83 & 4,3 & 0,49 \\
\hline 2 & 5554,98 & 10872,18 & 27180,45 & 315,06 & 1,96 & 0,43 \\
\hline 3 & 5637,48 & 10816,53 & 27041,34 & 322,91 & 2,52 & 0,43 \\
\hline 4 & 5431,5 & 10159,38 & 25638,46 & 338,06 & 1,47 & 0,43 \\
\hline 5 & 5789,02 & 11449,85 & 28624,63 & 326,83 & 5,35 & 0,43 \\
\hline 6 & 5417,47 & 10579,35 & 26448,38 & 321,65 & 1,56 & 0,43 \\
\hline 7 & 5554,98 & 10992,18 & 27480,46 & 311,69 & 2,06 & 0,43 \\
\hline 8 & 5678,98 & 11183,81 & 27959,52 & 303,88 & 1,85 & 0,43 \\
\hline 9 & 5623,98 & 10968,15 & 27420,37 & 333,62 & 4,96 & 0,43 \\
\hline 10 & 5651,5 & 10967,02 & 27417,55 & 314,7 & 2,24 & 0,43 \\
\hline 11 & 5445,02 & 10808,5 & 27021,25 & 312,79 & 1,49 & 0,43 \\
\hline 12 & 5706,499 & 11320,32 & 28300,79 & 326,14 & 2,62 & 0,43 \\
\hline 13 & 5445,02 & 11088,15 & 27720,37 & 326,73 & 1,73 & 0,45 \\
\hline 14 & 5706,499 & 11059,64 & 27649,1 & 325,21 & 1,37 & 0,41 \\
\hline 15 & 5445,02 & 11225,51 & 28063,78 & 319,72 & 1,12 & 0,47 \\
\hline 16 & 5706,499 & 11439,39 & 28598,47 & 323,9 & 2,6 & 0,43 \\
\hline 17 & 5445,02 & 11009 & 27522,5 & 332,56 & 2,16 & 0,45 \\
\hline 18 & 5706,499 & 11071,56 & 27678,89 & 320,08 & 0,42 & 0,43 \\
\hline
\end{tabular}

Podemos inferir que no existe una correlación lineal entre el incremento de la relación vidriado/opaco y los índices Bcal y Bref. Obtenemos diferentes configuraciones edilicias que mantienen un nivel de consumo energético equivalente, no afectado por la variación del porcentaje de vidrio en las fachadas -entre 43\% y $49 \%$ - sino por la configuración geométrica de la forma edilicia.

Figura 4. Relación \% vidrio/Bcal y Bref para las 18 alternativas Fuente: elaboración propia

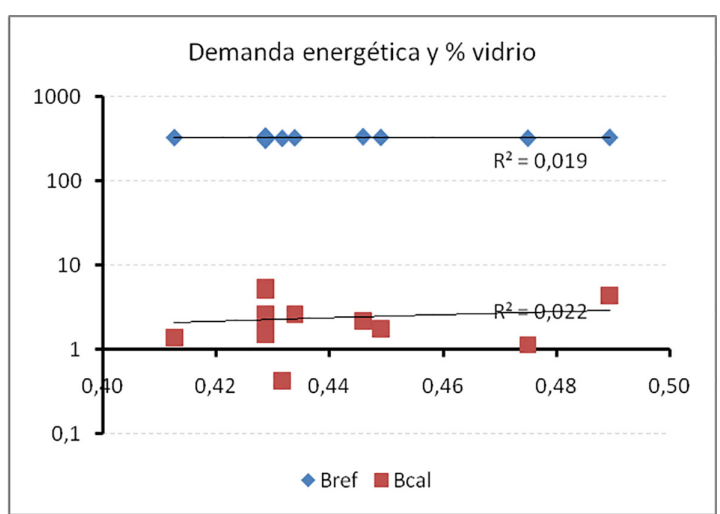

Se analiza la relación entre el porcentaje de vidrio para cada orientación respecto del total de la envolvente a fin de determinar su impacto en las cargas térmicas de calefacción y refrigeración. Cuando observamos la relación con Bref, (Figura 5) podemos observar que existe una mayor dependencia entre el incremento de la superficie vidriada al Oeste y el aumento del Bref, le sigue la orientación Este, pero no ocu- 
Figura 5. Relación entre el índice $B_{\text {ref }}$ y las superficies vidriadas por fachada Fuente: elaboración propia

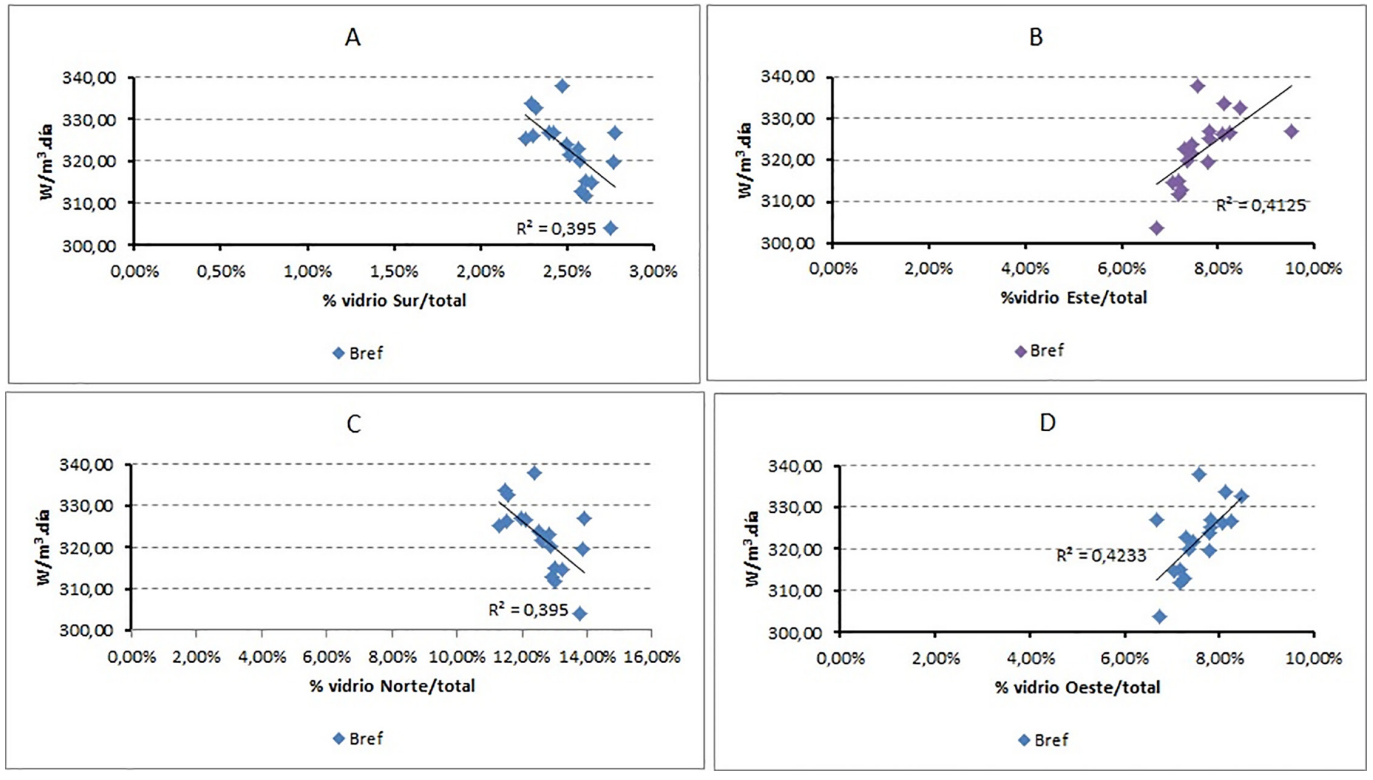

Figura 6. Relación entre el índice $B_{\text {cal }}$ l las superficies vidriadas por fachadasFuente: elaboración propia

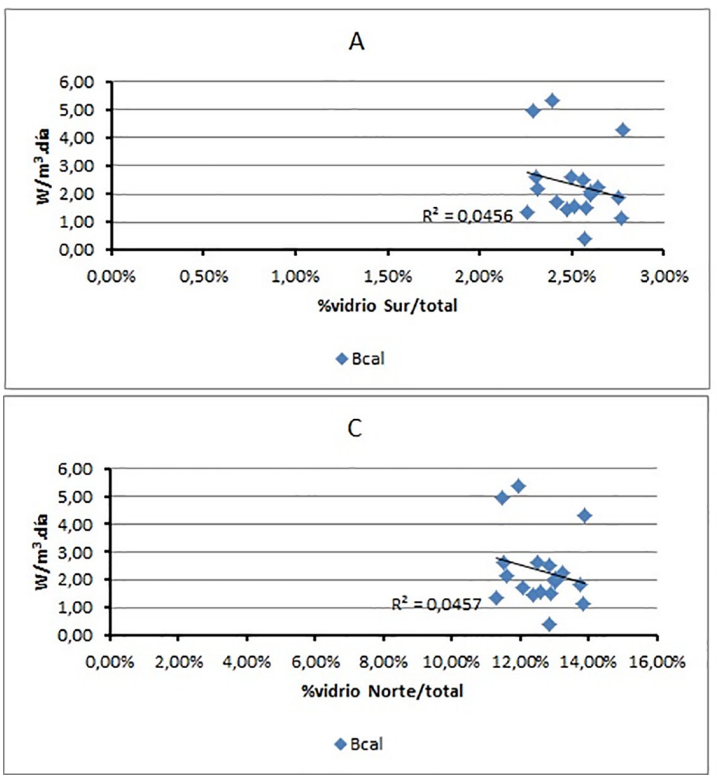

rre lo mismo para las orientaciones Norte y Sur. Esto ratifica la recomendación de contar con protección solar al Oeste (IRAM 11603).

Cuando relacionamos la misma variables con el índice Bcal, observamos una incidencia débil del vidriado para la fachada Este. Las demás orientaciones no muestran una relación entre superficies vidriadas y consumo energético (Figura 6).

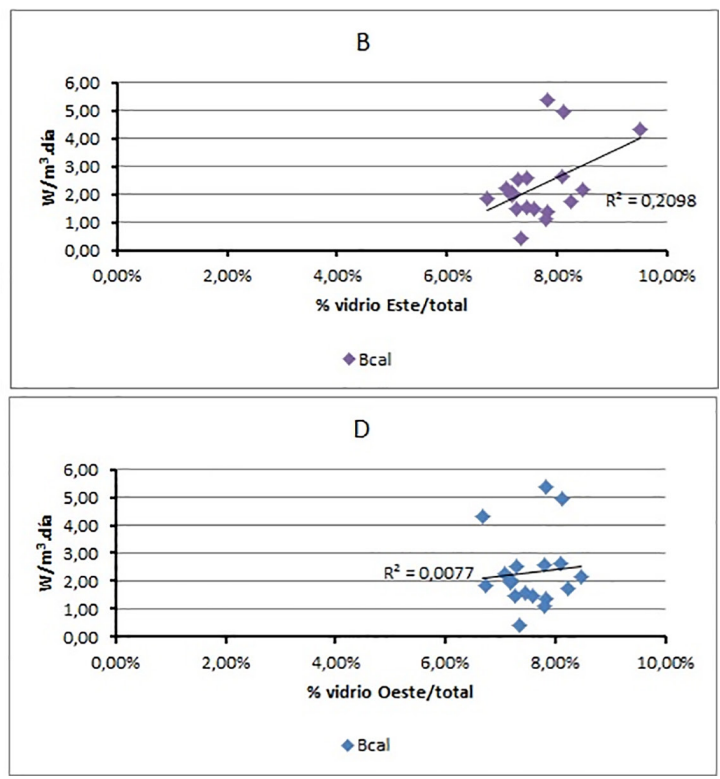

Al no existir una relación fuerte respecto de una orientación, decidimos operar sobre los cerramientos de todas las orientaciones —limitándonos a la alternativa 1-y buscando que el AG incremente la superficie vidriada sin incrementar el consumo energético.

Para alcanzar nuestro objetivo, habíamos mejorado los dispositivos de protección solar, con cortinas venecianas externas de aluminio, con 
Tabla 4. Consumo energético en la hora pico solar para un día típico de invierno Fuente: los autores

\begin{tabular}{|c|c|}
\hline Alternativas & $\mathrm{B}_{\text {cahora } 14}$ \\
\cline { 2 - 2 } & $\mathrm{W}$ \\
\hline 1 & $-8,26$ \\
\hline 2 & $-8,58$ \\
\hline 3 & $-8,72$ \\
\hline 4 & $-8,87$ \\
\hline 5 & $-8,30$ \\
\hline 6 & $-8,53$ \\
\hline 7 & $-8,48$ \\
\hline 8 & $-8,64$ \\
\hline 9 & $-8,34$ \\
\hline 10 & $-8,68$ \\
\hline 11 & $-8,44$ \\
\hline 12 & $-8,18$ \\
\hline 13 & $-8,26$ \\
\hline 14 & $-8,17$ \\
\hline 15 & $-8,68$ \\
\hline 16 & $-8,42$ \\
\hline 17 & $-8,22$ \\
\hline 18 & $-8,62$ \\
\hline
\end{tabular}

un factor de protección solar (Fs) igual a 0.10 . De la observación de la Tabla 4, deducimos que el Bcal negativo nos está indicando que existe un exceso de calor que provocaría sobrecalentamiento y provocaría el encendido del equipo de refrigeración en la hora 14. Esto es válido para las dieciocho alternativas iniciales.

Iteramos con el AG aplicado a los porcentajes de vidrio de todas las fachadas, manteniendo fija la morfología (alternativa 1) y aumentando el Fs de 0,35 a 0,1. El Bref se mantiene por debajo del correspondiente a la alternativa 1 . Obtenemos alternativas con mayor superficie de elementos vidriados, y conseguimos una disminución del sobrecalentamiento en la hora 14 para el día típico de invierno (Tabla 5), disminuyendo asimismo el consumo de energía en verano. La disminución de la ganancia solar en invierno provoca un incremento del consumo en calefacción, pero aún así, se mantiene muy por debajo del de refrigeración. El aumentodel consumo de calefacción, se contrarresta con un ahorro del equipo de refrigeración que debía encenderse para recuperar la temperatura de confort $\left(25^{\mathrm{a}} \mathrm{C}\right)$ (Tabla 5$)$.

La mayor aislación de los cerramientos redunda en un mejor desempeño energético ya que disminuye la brecha entre la transmitancia de los elementos opacos y los vidriados.
Tabla 5. Optimización de la superficie vidriada de la alternativa 1Fuente: elaboración propia

\begin{tabular}{|c|c|c|c|c|c|c|c|c|c|c|}
\hline $\begin{array}{c}\text { Alternati } \\
\text { va }\end{array}$ & $F_{s}$ & $\mathrm{~B}_{\text {cal }}$ & $\begin{array}{c}\text { B }_{\text {cahora }} \\
14\end{array}$ & $\mathrm{~B}_{\text {ref }}$ & $\begin{array}{l}\% v i \\
d N\end{array}$ & & $\begin{array}{l}\text { \%vi } \\
d E\end{array}$ & $\begin{array}{l}\text { \%vi } \\
\text { do }\end{array}$ & $\begin{array}{c}\text { \%vid/envolv } \\
\text { ente }\end{array}$ & $\begin{array}{c}\text { \%vid/o } \\
\text { p. }\end{array}$ \\
\hline 1 & $\begin{array}{c}0,3 \\
5\end{array}$ & 4,3 & $-8,26$ & $\begin{array}{c}326, \\
83\end{array}$ & 50 & 10 & 40 & 30 & 0,32 & 0,49 \\
\hline 1.1 & 0,1 & $\begin{array}{c}73,0 \\
3\end{array}$ & 0,35 & $\begin{array}{c}13,8 \\
5\end{array}$ & 50 & 10 & 40 & 30 & 0,32 & 0,49 \\
\hline 1.2 & 0,1 & $\begin{array}{c}156, \\
55\end{array}$ & 1,08 & $\begin{array}{c}327, \\
14\end{array}$ & 60 & 87 & 41 & 56 & 0,62 & 1,63 \\
\hline 1.3 & 0,1 & $\begin{array}{c}170, \\
83\end{array}$ & 1,84 & $\begin{array}{c}315, \\
02\end{array}$ & 75 & 69 & 49 & 64 & 0,65 & 1,86 \\
\hline 1.4 & 0,1 & $\begin{array}{c}205 \\
9\end{array}$ & 2,67 & $\begin{array}{c}315, \\
04\end{array}$ & \begin{tabular}{|l|}
91 \\
\end{tabular} & 35 & 87 & 89 & 0,74 & 2,85 \\
\hline 1.5 & 0,1 & $\begin{array}{c}160, \\
22\end{array}$ & 1,33 & $\begin{array}{c}316, \\
69\end{array}$ & 71 & 54 & 38 & 81 & 0,61 & 1,56 \\
\hline 1.6 & 0,1 & $\begin{array}{c}223 \\
54\end{array}$ & 3,94 & $\begin{array}{c}317, \\
57\end{array}$ & 100 & 94 & 94 & 33 & 0,82 & 4,56 \\
\hline
\end{tabular}

Al relacionar los datos de $B_{\text {cal }}$, $B_{\text {ref }}$ con los porcentajes de superficies vidriadas respecto de las superficies opacas, encontramos que existe unacorrelación positiva para la condición de invierno (Figura7). No sucede lo mismo con la condición de verano donde a igual demanda, corresponden notables variaciones en el porcentaje de superficies vidriadas de las alternativas 1.2 a 1.6. Esto se debe a que el AG estuvo condicionado en mantener el Bref pero buscó alternativas de mayor superficie vidriada en la orientación Sur, donde pasó de un 10\% hasta un 94\%. Esto puede observarse claramente para la alternativa 1.6 donde se incrementó la superficie vidriada al Norte: de $50 \%$ a $100 \%$, al Sur: de $10 \%$ a $94 \%$, al Este: de $40 \%$ a $94 \%$ pero se mantuvo la fachada Oeste: de 30\% a 33\%. Se pasó así de tener un 32\% de vidrio/opaco a un $82 \%$ de vidrio/opaco, con una demandasimilar en verano, aumentando sólo la protección solar. Las otras alternativas permiten observar cómo se fueron balanceando los incrementos de vidriado en todas las orientaciones, entre $71 \%$ y $84 \%$. Los rangos de porcentaje de vidrio se ampliaron para todas las orientaciones de $0 \%$ a 100\%, mientras que en las alternativas iniciales se encontraban fijos en $50 \% \mathrm{~N}, 40 \% \mathrm{E}$, 10\%S y30\% O. Esto permitió que el AG ampliara su espacio de búsqueda y combinara todas las orientaciones entre $61 \%$ y $82 \%$ de relación vidriado/opaco. Al incrementar el vidriado al 
Figura 7. Relación Bcal-Bref y \% vidrio/opaco Fuente: los autores

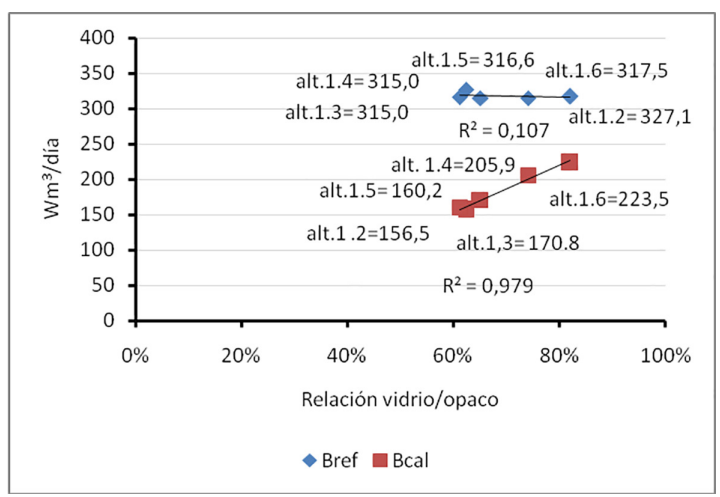

Sur, aumentaron las pérdidas en invierno. Los porcentajes iniciales estaban orientados a una minimización de las pérdidas de invierno, de acuerdo a lo recomendado por la norma IRAM 11603, en cambio, las alternativas obtenidas con el AG, no están restringidas por esas recomendaciones.

En el gráfico de lafigura 7 , no se considera la alternativa 1.1- que difiere de la alternativa 1 en la aislación de las ventanas pero mantiene idénticos porcentajes de superficie vidriadadebido a la consecuente y abrupta disminución de Bref (13,5 W/m3día invierno).

Cabe señalar que, si se simulase la intervención del usuario en el accionamiento de las cortinas, podrían obtenerse consumos de calefacción muy inferiores al aprovechar la ganancia solar durante el día.

\section{Conclusiones}

Los resultados del trabajo muestranque las decisiones proyectuales pueden ser contrarias a la intuición, ya que los resultados de los índices de demanda energéticaexhiben alternativas no previstas. La complejidad de la performance energética muestra que no existe linealidad entre las estrategias de proyecto y las demandas energéticas, en la instancia de la modelización. No puede contrastarse con casos reales, al tratarse de una simulación.

Los modelos paramétricos permiten la evaluación del desempeño energético deformas edilicias en tiempo real. Los rangos de datos de entrada son regulados manualmente por el diseñador u optimizados mediante el uso de algoritmos genéticos. En este caso, utilizamos el factor solar como variable, pero podríamos haber iterado sobre cualquier otro input de nuestro interés (infiltración, materialidad de la envolvente, ocupación, costos, etc.). Para disminuir la demanda energética de calefacción y refrigeración, utilizamos la opción de incrementar la aislación térmica de las aberturas, sin aumentar la aislación de los muros.

Los índices bioclimáticos de calefacción Bcal y refrigeración Bref muestran el desempeño energético para días típicos de invierno y verano respectivamente, en estado estacionario. Su aporte es la incorporación de las ganancias solares, las ganancias internas, la determinación de la infiltración a partir de datos climáticos, yla permeabilidad de ventanas y su grado de exposición al viento para la condición de inviernomediante el cómputo horario del consumo de energía- para ambas situaciones. Una evolución lógica es incorporar las variables costo y emisiones GEI.

Esta herramienta ofrece un desarrollo muy amplio para la incorporación de variables y su optimización en el campo del diseño arquitectónico energéticamente eficiente para la mitigación del Cambio Climático. Muestra su valor como herramienta de pre-proyecto sustentable.

\section{Bibliografía}

BEN Balance Energético Nacional, Ministerio de Economía de la República Argentina http://www.energia.gov.ar/ contenidos/verpagina.php?idpagina $=3366$ 
Camporeale, P., (2012). El uso de algoritmos genéticos en el diseño paramétrico de edificios energéticamente eficientes: el coeficiente G y el consumo anual de energía, Actas del XII Congreso Iberoamericano de Energía Solar (CIES), Vigo, España

Camporeale, P. y Czajkowski, J.(2015). Índice Bioambiental de Consumo Energético en Edificios aplicado a un Modelo de Diseño Paramétrico- Actas del II Congreso Internacional de Construcción Sostenible y Soluciones Eco-eficientes- 25 al 27 de mayo-de 2015- págs. 629 y ss. ISBN 978-84-617-3964-6.Sevilla- España

Goldberg, D., (1997). 'Some applications of Genetic Algorithms' in 'Genetic Algorithms in Search, Optimization and Machine Learning', Addison-Wesley, MA, USA, pp. 88-145.

Gonzalo, G. E., (1998). Manual de arquitectura bioclimática. Tucumán: Edicióndelautor.

Iqbal, Imran etals (2007). Parametric analysis of alternative energy conservation measures in an office building in hot and humid climate Building and Environment, Volume 42, Issue 5, May 2007, Pages 2166-2177

Leach, N etals, (2004). 'Design by Algorithm' in 'Digital tectonics', Wiley-Academy, UK, pp. 79-87.

Mitchell, M. (1998), 'Implementing a genetic algorithm' in 'An Introduction to Genetic Algorithms', The MIT Press, MA, USA, pp. 154-179

Norma IRAM 11507-4 (2012).Carpintería de obra y fachadas integrales livianas. Ventanas exteriores Parte 4.Requisitos complementarios. Aislación térmica. . Instituto Argentino de Normalización. Buenos Aires.

Norma IRAM 11601:(2002). Aislamiento térmico de edificios, métodos de cálculo. Propiedades térmicas de los componentes y elementos de construcción en régimen estacionario. Instituto Argentino de Normalización. Buenos Aires.

Norma IRAM 11603(1996). Acondicionamiento térmico de edificios. Clasificación bioambiental de la República Argentina. Instituto Argentino de Normalización. Buenos Aires.

Norma IRAM 11604 (2001). Aislamiento térmico de edificios. Verificación de sus condiciones higrotérmicas. Ahorro de energía en calefacción. Coeficiente volumétrico $\mathrm{G}$ de pérdidas de calor. Cálculo y valores límites- Instituto Argentino de Normalización y Certificación- $2^{\circ}$ ed.-Buenos Aires

Norma IRAM 11605 (1996). Acondicionamiento térmico de edificios, condiciones de habitabilidad en edificios. Valores máximos de transmitancia térmica en cerramientos opacos. Instituto Argentino de Normalización. Buenos Aires.

Norma IRAM 116591 y 2-(2004). Aislamiento térmico de edificios. Verificación de sus condiciones higrotérmicas. Ahorro de energía en refrigeración. Parte 1: Vocabulario, definiciones, tablas y datos para determinar la carga térmica de refrigeración- Parte 2: Edificios para viviendas- Instituto Argentino de Normalización y Certificación-Buenos Aires

Peronato, g. etals. (2015). A parametric design based methodology to visualize building performance at the neighborhood scale- Infoscience- Écolepolytechniquefédérale de Lausanne EPFL

Sadineni,Suresh B.,MadalaSrikanth, Boehm Robert F.- (2011) Passive building energy savings: A review of building envelope components- Renewable and Sustainable Energy Reviews 15 3617-3631

Terzidis, K. (2006). Algorithmic Architecture. Oxford: Architectural Press.

Russell, S. et al, (2010).Beyond classical search in Artificial Intelligence, A Modern Approach, 3rd ed., Prentice-Hall Pearson Education Inc., NJ, USA, pp. 125-128. 